\title{
Expression Profiles of Wnt Genes during Neural Differentiation of Mouse Embryonic Stem Cells
}

\begin{abstract}
The Wnt family of secreted signaling proteins regulates many aspects of animal development and the behavior of several types of stem cells, including embryonic stem (ES) cells. Activation of canonical Wnt signaling has been shown to either inhibit or promote the differentiation of ES cells into neurons, depending on the stage of differentiation. Here, we describe the expression of all 19 mouse Wnt genes during this process. Using the wellestablished retinoic acid induction protocol we found that all Wnt genes except Wnt8b are expressed as ES cells differentiate into neurons, many of them in dynamic patterns. The expression pattern of $12 \mathrm{Wnt}$ genes was analyzed quantitatively at 2-day intervals throughout neural differentiation, showing that multiple Wnt genes are expressed at each stage. A large proportion of these, including both canonical and noncanonical Wnts, are expressed at highest levels during later stages of differentiation. The complexity of the patterns observed indicates that disentangling specific roles for individual Wnt genes in the differentiation process will be a significant challenge.
\end{abstract}

